\title{
A high-carbohydrate diet induces greater inflammation than a high-fat diet in mouse skeletal muscle
}

\author{
M.M. Antunes (iD ${ }^{1}$, G. Godoy (iD ${ }^{1}$, C.B. de Almeida-Souza ${ }^{1}{ }^{1}$, B.A. da Rocha ${ }^{1}{ }^{1}$, L.G. da Silva-Santi(i) ${ }^{1}$,

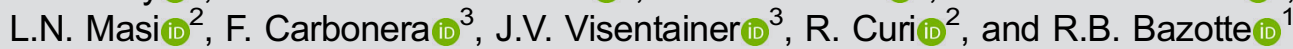 \\ ${ }^{1}$ Departamento de Farmacologia e Terapêutica, Universidade Estadual de Maringá, Maringá, PR, Brasil \\ ${ }^{2}$ Programa de Pós-Graduação Interdisciplinar em Ciências da Saúde, Universidade Cruzeiro do Sul, São Paulo, SP, Brasil \\ ${ }^{3}$ Departmento de Química, Universidade Estadual de Maringá, Maringá, PR, Brasil
}

\begin{abstract}
We previously reported that both the high-carbohydrate diet (HCD) and high-fat diet (HFD) given for two months promote lipid deposition and inflammation in the liver and brain of mice. The results obtained indicate a tissue-specific response to both diets. Herein, we compared the effects of HCD and HFD on fatty acid (FA) composition and inflammation in the gastrocnemius muscle. Male Swiss mice were fed with HCD or HFD for 1 or 2 months. Saturated FA (SFA), monounsaturated FA (MUFA), n-3 polyunsaturated FA ( $n-3$ PUFA), and n-6 PUFA were quantified. The activities of stearoyl-CoA desaturase 1 (SCD-1), $\Delta-6$ desaturase (D6D), elongase 6, and de novo lipogenesis (DNL) were estimated. As for indicators of the inflammatory tissue state, we measured myeloperoxidase (MPO) activity and gene expression of F4/80, tumor necrosis factor- $\alpha$ (TNF- $\alpha$ ), interleukin (IL)-4, IL-6, and IL-10. The HCD led to a lower deposition of SFA, MUFA, n-3 PUFA, and n-6 PUFA compared to HFD. However, the HCD increased arachidonic acid levels, SFA/n-3 PUFA ratio, DNL, SCD-1, D6D, and MPO activities, and expression of IL-6, contrasting with the general idea that increased lipid deposition is associated with more intense inflammation. The HCD was more potent to induce skeletal muscle inflammation than the HFD, regardless of the lower lipid accumulation.
\end{abstract}

Key words: Saturated fatty acids; Monounsaturated fatty acids; Polyunsaturated fatty acids; n-6/n-3 PUFA ratio; SFA/n-3 PUFA ratio

\section{Introduction}

Both blood fatty acids (FA) and tissue-triacylglycerolderived FA are sources of ATP for skeletal muscle contraction (1). Lipids stored in skeletal muscles play an important role as an energy supply during physical exercise. However, abnormal lipid deposition in the skeletal muscles of sedentary and obese individuals is associated with inflammation, insulin resistance, type 2 diabetes, cardiovascular diseases, and myopathies $(2,3)$.

Diet-induced obesity promotes insulin resistance $(4,5)$, lipid accumulation $(6,7)$, and inflammation $(8,9)$ in skeletal muscle. The regulation of skeletal muscle FA composition is not fully understood; however, it markedly changes with dietary macronutrient composition. Skeletal muscle FA composition varies according to the proportion of the FA present in the diet $(10,11)$. These studies, however, do not differentiate the effects of macronutrient composition from those caused by obesity.

As described in other reports $(8,12,13)$, we previously demonstrated that a high-carbohydrate diet (HCD) given for 2 months leads to a similar body weight gain compared with a high-fat diet (HFD) in Swiss mice $(14,15)$, allowing the evaluation of diet-induced changes in FA composition and inflammatory markers without the influence of obesity. In these studies, we reported changes in FA composition and inflammatory markers in the liver (14) and brain (15). Herein, we extended the previous studies to skeletal (gastrocnemius) muscle of male Swiss mice.

\section{Material and Methods}

\section{Animals and experimental design}

The Scientific Advisory Committee on Animal Care of the State University of Maringá (protocol No. 3105210717) approved the experimental procedures of the present study, following the International Guidelines for the Use and Care of Laboratory Animals.

Weaned mice received standard rodent chow (Nuvilab $^{\mathrm{TM}}$, Brazil) $(53.3 \%$ carbohydrates, $22 \%$ proteins, and $4.5 \%$ lipids). The starting point (Time 0 ) was considered to be when the mice reached six weeks of age (about $33 \mathrm{~g}$ body weight). They were then randomly divided into groups that received $\mathrm{HCD}(73.8 \%$ carbohydrates, $14.2 \%$ proteins, 


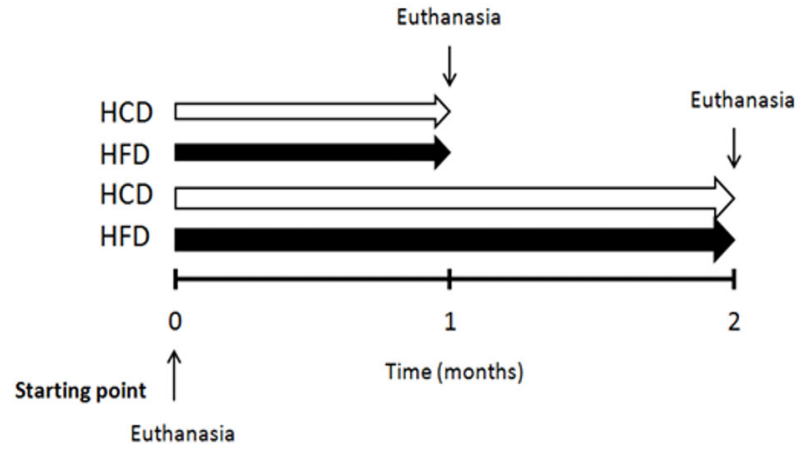

Figure 1. Experimental design. All mice received standard rodent chow before starting (time 0 ) and a high-fat diet (HFD) or a highcarbohydrate diet (HCD) was administered for 1 or 2 months.

and $4 \%$ lipids) or HFD (36.5\% carbohydrates, $20.3 \%$ proteins, and $35.2 \%$ lipids) for 1 or 2 months (Figure 1 ). Specific nutrients and FA composition of the diets (HCD or HFD) are described in our previous publications $(14,16)$. All mice had free access to food and water. We recorded caloric intake and body weight throughout the study.

Overnight-fasted mice (from 17:00 to 08:00), as described in our previous studies $(9,14-17)$, were then euthanized by decapitation. Gastrocnemius muscle from both legs was removed, frozen in liquid nitrogen, and stored at $-80^{\circ} \mathrm{C}$ until analysis.

\section{Fatty acids composition}

We used the method of Bligh and Dyer (18) on a reduced-scale to extract total lipids from the gastrocnemius muscle. FA methyl esters (FAME) were prepared by ultrasound-assisted total lipid methylation, as described by Santos et al. (19). FAME was separated by gas chromatography. Retention times and peak areas were determined using the Chrom-Quest ${ }^{\mathrm{TM}}$ software (Thermo Scientific $^{\mathrm{TM}}$, USA). FA contents in the muscles were reported as $\mathrm{mg} / \mathrm{g}$ of total fat.

Estimated de novo lipogenesis (DNL) and activities of stearoyl-CoA desaturase 1 (SCD-1), $\Delta-6$ desaturase (D6D), and elongase 6

DNL and activities of SCD-1, D6D, and elongase 6 were estimated using the product/precursor ratios of individual FA in lipid esters as follows: $D N L$ as the ratio of $16: 0 / 18: 2 n-6$; SCD-1 activity index as the ratio of $16: 1 n-7 /$ $16: 0$; D6D activity index as the ratio of $18: 3 n-6 / 18: 2 n-6$; and elongase 6 activity index as the ratio of 18:0/16:0.

\section{Determination of myeloperoxidase (MPO) activity}

Gastrocnemius muscles of mice fed with HCD or HFD for 2 months were homogenized in phosphate-buffered saline (PBS), and the homogenate was stirred in a vortex and centrifuged $\left(500 \mathrm{~g}, 4^{\circ} \mathrm{C}\right)$ for $5 \mathrm{~min}$. The activity of MPO was measured in tissue supernatants $(10 \mu \mathrm{L})$ in triplicate.
PBS $(0.2 \mathrm{~mL})$ containing o-dianisidine dihydrochloride (4.2 $\mathrm{mg})$, double-distilled water $(22.5 \mathrm{~mL})$, potassium phosphate buffer $(2.5 \mathrm{~mL}, \mathrm{pH}=6)$, and $\mathrm{H}_{2} \mathrm{O}_{2}(10 \mu \mathrm{L}, 1 \%)$ was also added. The enzyme reaction was stopped by the addition of $30 \mu \mathrm{L}$ sodium acetate $(2.23 \mathrm{~g}$ in $20 \mathrm{~mL}$ of double-distilled water). MPO activity was determined at $460 \mathrm{~nm}$, using a microplate spectrophotometer (Asys Expert Plus, Biochrom, UK), and is reported as absorbance $(\mathrm{Ab})$.

\section{Gene expression measurement}

$\mathrm{F} 4 / 80$, tumor necrosis factor- $\alpha(\mathrm{TNF}-\alpha)$, interleukin (IL)-6, IL-4, and IL-10 mRNA expressions were measured in the gastrocnemius muscle of mice fed with HCD or HFD for 2 months. The gastrocnemius muscles $(20 \mathrm{mg})$ were powdered in liquid nitrogen and total RNA was extracted using Trizol reagent (Invitrogen Life Technologies, USA). Reverse transcription to cDNA was performed using the High-Capacity cDNA kit (Applied Biosystems, USA). Gene expression was evaluated by real-time PCR using SYBR Green as the fluorescent dye (Invitrogen Life Technologies).

The quantification of gene expression was performed using the comparative $\mathrm{Ct}$ method (Ct: threshold cycle, the cycle number in which the PCR product reaches the detection threshold). $\beta 2$-microglobulin gene $(\beta 2 \mathrm{~m})$ expression was used as a reference.

The primer sequences were: F4/80, NM_010130.4, sense CCTGAACATGCAACCTGCCAC, antisense GGG CATGAGCAGBCTGTAGGATC; TNF- $\alpha$, NM_001278601.1, sense TCTTCTCATTCCTGCTTGTGGC, antisense CACT TGGTGGTTTGCTACGACG; IL-6, NM_001314054.1, sense GGTAGCATCCATCATTTCTTTG, antisense CGGAGAGGA GACTTCACAAGAG; IL-4, NM_021283.2, sense CCATATC CACGGATGCGACA, antisense CTGTGGTGTTCTTCGTT GCTG; IL-10, NM_010548.2, sense TGCCAAGCCTTATCG GAAATG, antisense AAATCGATGACAGCGCCTCAG.

\section{Statistical analysis}

The results are reported as means \pm SE. One-way ANOVA followed by the Tukey's post-test was used to evaluate differences between 0,1 , and 2 months. The Student's t-test was used to assess differences between the HCD and HFD groups. Statistical analyses were performed using GraphPad Prism 5.0 software (USA). A P-value $<0.05$ indicated statistical significance.

\section{Results}

\section{Caloric intake, body weight, and gastrocnemius muscle weights}

The daily caloric intake was $32.6 \pm 5.8 \mathrm{kcal} /$ day for HCD group and $22.6 \pm 2.0 \mathrm{kcal} /$ day for HFD group after 1 month of diet intervention, and $29.4 \pm 2.0 \mathrm{kcal} / \mathrm{day}$ for HCD group and $20.9 \pm 0.5 \mathrm{kcal} /$ day for HFD group after 2 months. 

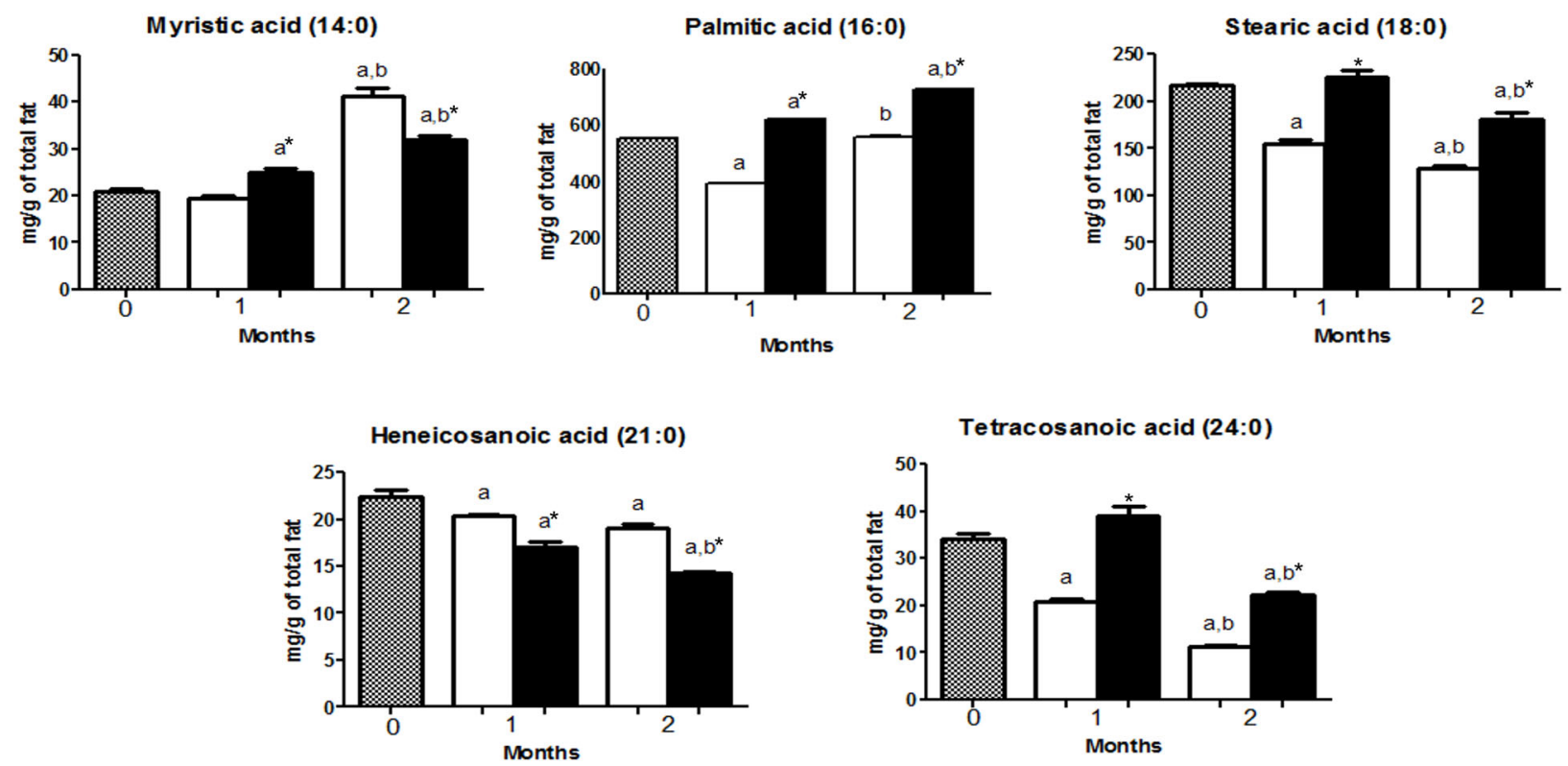

Figure 2. Saturated fatty acid (SFA) composition in the gastrocnemius muscle of mice at 0 (before starting the diets, gray bars) or fed with a high-carbohydrate diet (HCD, white bars) or high-fat diet (HFD, black bars) for 1 or 2 months. The concentrations of SFA are reported as means $\pm \mathrm{SE}$. ${ }^{*} \mathrm{P}<0.05$ compared to $\mathrm{HCD}$ group (Student's $t$-test). ${ }^{\mathrm{a}} \mathrm{P}<0.05$ compared to time 0 ; ${ }^{\mathrm{b}} \mathrm{P}<0.05$ compared to 1 month (one-way ANOVA).

The final body weight was $45.8 \pm 1.4 \mathrm{~g}$ for $\mathrm{HCD}$ group and $45.0 \pm 2.2 \mathrm{~g}$ for HFD group after 1 month, and $51.0 \pm 1.9 \mathrm{~g}$ for $\mathrm{HCD}$ group and $50.6 \pm 1.5 \mathrm{~g}$ for HFD group after 2 months.

The gastrocnemius muscle weights were $0.47 \pm 0.01 \mathrm{~g}$ in the HCD group and $0.45 \pm 0.01 \mathrm{~g}$ in the HFD group, 2 months after starting the diets.

\section{FA composition, estimated DNL, and activities of SCD-1, D6D, and elongase 6}

HCD and HFD mice had a higher content of palmitic acid (16:0), oleic acid (18:1 n-9), and linoleic acid (18:2n-6) compared with other FA (Figures 2, 3, and 4).

Heneicosanoic acid (21:0) and palmitoleic acid $(16: 1 n-7)$ were increased $(P<0.05)$ in the HCD group compared to HFD group, after 1 and 2 months. Myristic acid (14:0), vaccenic acid (18:1n-7), arachidonic acid $(20: 4 n-6)$, docosapentaenoic acid (22:5n-6), and eicosapentaenoic acid $(20: 5 n-3)$ were higher $(P<0.05)$ in the HFD only after 1 month of diet intervention but did not differ (HFD vs HCD) after 2 months (Figures 2, 3, and 4).

Palmitic acid (16:0), stearic acid (18:0), tetracosanoic acid (24:0), 7-hexadecanoic acid (16:1n-9), oleic acid $(18: 1 n-9)$, linoleic acid (18:2n-6), docosatetraenoic acid $(22: 4 n-6), \alpha$-linolenic acid (18:3n-3), and docosahexaenoic acid $(22: 6 n-3)$ were higher $(P<0.05)$ in the HFD group compared to HCD group after 1 and 2 months of diet intervention. $\gamma$-linolenic acid (18:3n-6) was higher
$(P<0.05)$ only after 1 month and did not differ between groups after 2 months (Figures 2 to 5 ).

We observed an increase $(P<0.05)$ in myristic acid (14:0), palmitoleic acid (16:1n-7), 7-hexadecanoic acid $(16: 1 n-9)$, vaccenic acid (18:1n-7), and oleic acid (18:1n-9) content (Time 0 vs 2 months) in both HCD and HFD groups (Figures 2 and 3 ).

The contents of stearic acid (18:0), heneicosanoic acid (21:0), tetracoisanoic acid (24:0), arachidonic acid (20:4n-6), docosatetraenoic acid (22:4n-6), docosapentaenoic acid (22:5n-6), eicosapentaenoic acid (20:5n-3), and docosahexanoic acid (22:6n-3) decreased in both HFD and HCD after 2 months (Figures 2, 4, and 5).

The HCD group had lower $(P<0.05)$ deposition of saturated fatty acids (SFAs), monounsaturated fatty acids (MUFAs), $n-6$ polyunsaturated fatty acids (PUFAs), and $n-3$ PUFAs compared to HFD after 1 and 2 months (Figure 6).

Total fat accumulation, calculated by the sum of all FA, was more significant for the HFD group after 1 and 2 months of starting the diets (HFD vs HCD groups) (Figure 6).

The HCD group exhibited a higher $(P<0.05)$ SFA/n-3 ratio than the HFD group 1 and 2 months after starting the diets. However, there was no difference in the $n-6 / n-3$ ratio (Figure 6).

DNL (16:0/18:2n-6), SCD-1 (16:1n-7/16:0), and D6D $(18: 3 n-6 / 18: 2 n-6)$ activities were higher $(P<0.05)$ in HCD compared to HFD group after 1 or 2 months of diet interventions. Elongase 6 activity did not differ between groups after 1 or 2 months of diet interventions (Figure 7). 

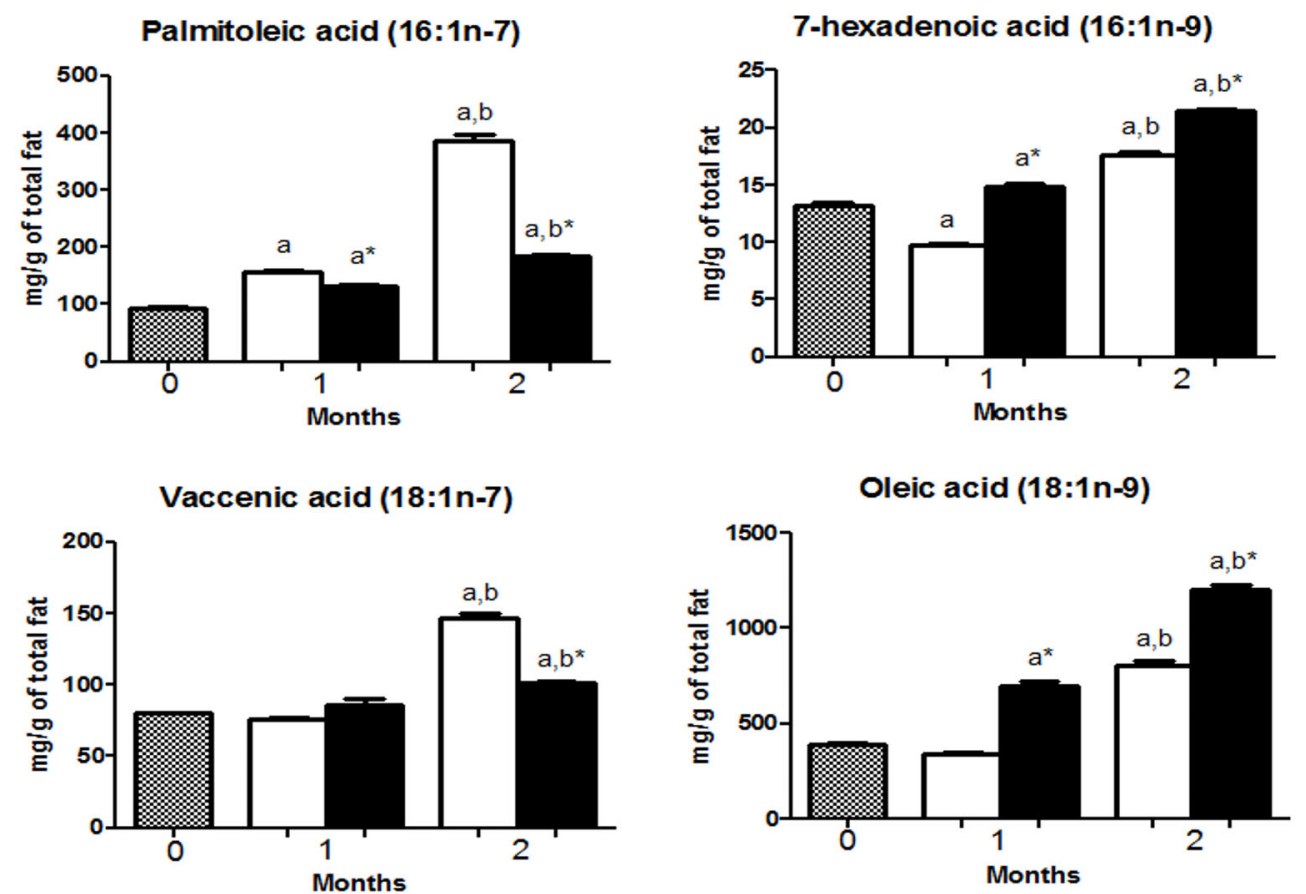

Figure 3. Monounsaturated fatty acids (MUFAs) composition in the gastrocnemius muscle of mice at 0 (before starting the diets, gray bars) or fed with a high-carbohydrate diet (HCD, white bars) or high-fat diet (HFD, black bars) for 1 or 2 months. The concentrations of MUFAs are reported as means $\pm \mathrm{SE}$. ${ }^{*} \mathrm{P}<0.05$ compared to HCD group (Student's $t$-test). ${ }^{\mathrm{a}} \mathrm{P}<0.05$ compared to time 0 ; ${ }^{b} \mathrm{P}<0.05$ compared to 1 month (one-way ANOVA).
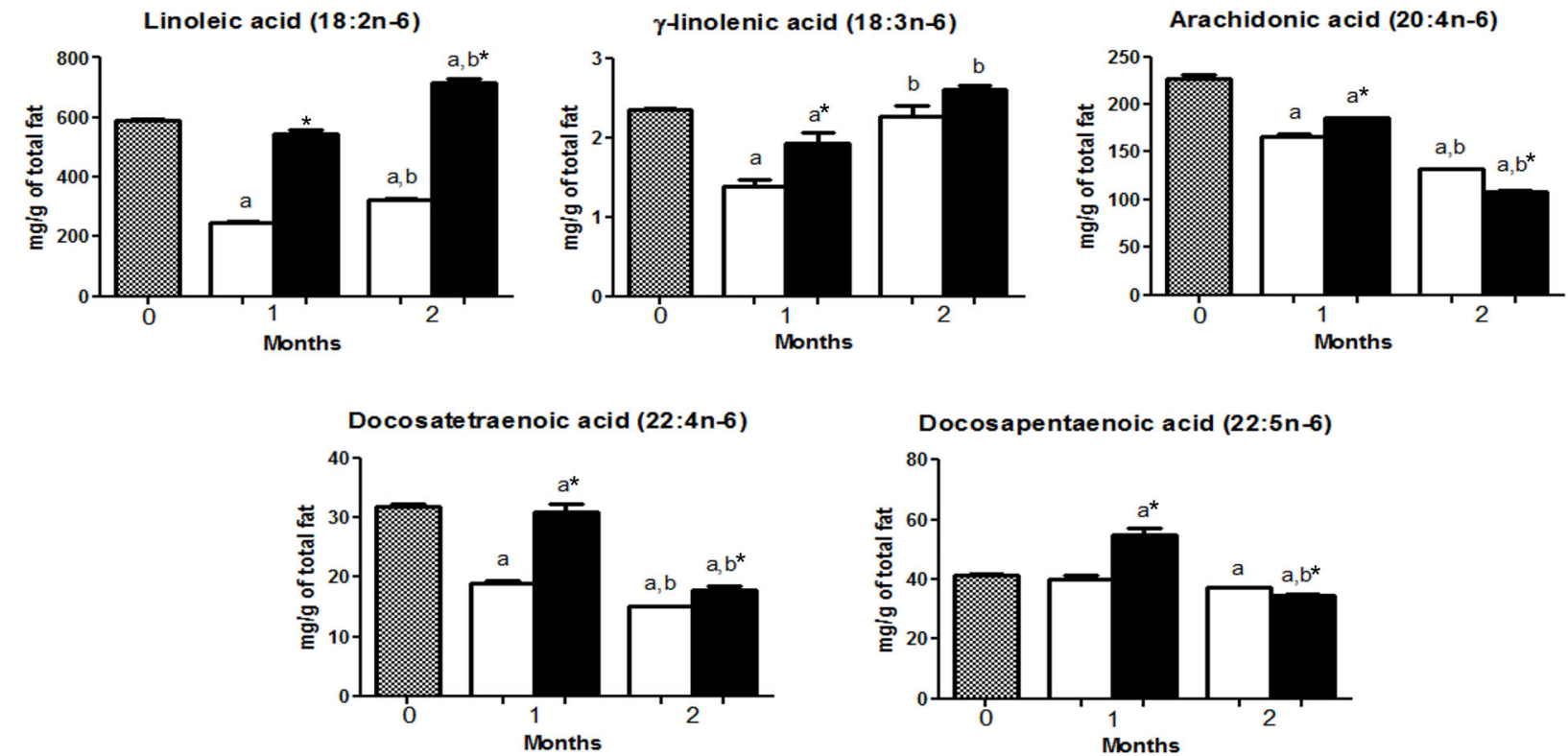

Figure 4. Polyunsaturated n-6 fatty acid (n-6 PUFA) composition in the gastrocnemius muscle of mice at 0 (before starting the diets, gray bars) or fed with a high-carbohydrate diet (HCD, white bars) or high-fat diet (HFD, black bars) for 1 or 2 months. The levels of $\mathrm{n}-6$ PUFA are reported as means \pm SE. ${ }^{*} \mathrm{P}<0.05$ compared to HCD group (Student's $t$-test). ${ }^{\mathrm{a}} \mathrm{P}<0.05$ compared to time 0 ; ${ }^{\text {b }} \mathrm{P}<0.05$ compared to 1 month (one-way ANOVA). 

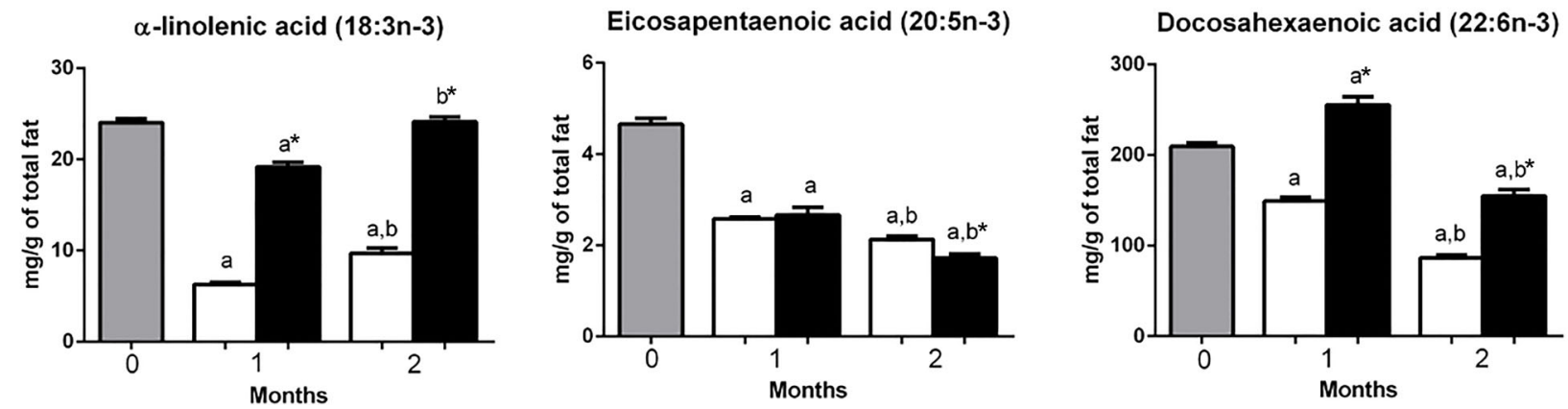

Figure 5. Polyunsaturated n-3 fatty acids (n-3 PUFAs) composition in the gastrocnemius muscle of mice at 0 (before starting the diets, gray bars) or fed with a high-carbohydrate diet (HCD, white bars) or high-fat diet (HFD, black bars) for 1 or 2 months. The contents of $n-3$ PUFAs are reported as means $\pm \mathrm{SE}$. ${ }^{*} \mathrm{P}<0.05$ compared to HCD group (Student's t-test). ${ }^{\mathrm{a}} \mathrm{P}<0.05$ compared to time 0 ; ${ }^{\mathrm{b}} \mathrm{P}<0.05$ compared to 1 month (one-way ANOVA).
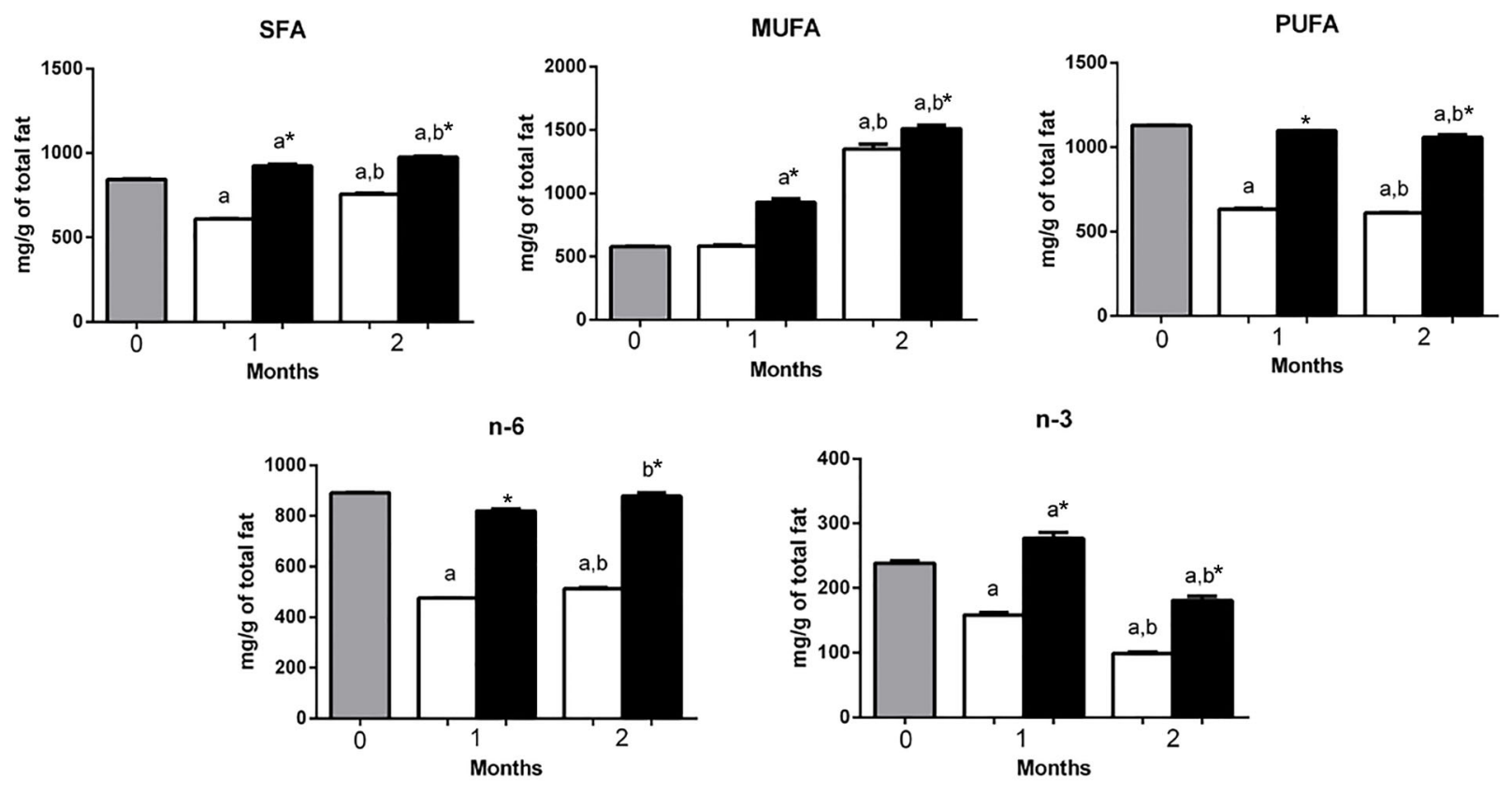

$\mathrm{n}-6 / \mathrm{n}-3$

SFA/n-3
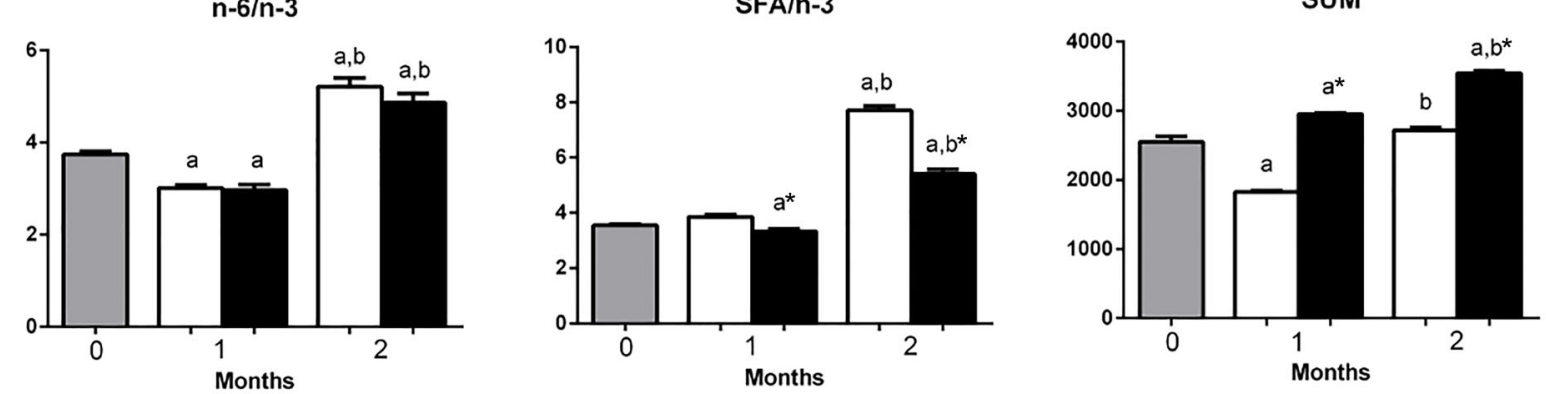

Figure 6. Fatty acid family composition and the $n-6 / n-3$ and SFA/n-3 ratios in the gastrocnemius muscle of mice at 0 (before starting the diets, gray bars) or fed with a high-carbohydrate diet (HCD, white bars) or high-fat diet (HFD, black bars) for 1 or 2 months. Results are reported as means $\pm \mathrm{SE}$. ${ }^{*} \mathrm{P}<0.05$ compared to $\mathrm{HCD}$ group (Student's $t$-test). ${ }^{\mathrm{a}} \mathrm{P}<0.05$ compared to time 0 ; ${ }^{\text {b }} \mathrm{P}<0.05$ compared to 1 month (one-way ANOVA). SFA: total saturated fatty acids; MUFA: total monounsaturated fatty acids; PUFA: total polyunsaturated fatty acids; SUM: sum of all fatty acids evaluated. 
SCD-1 (16:0)

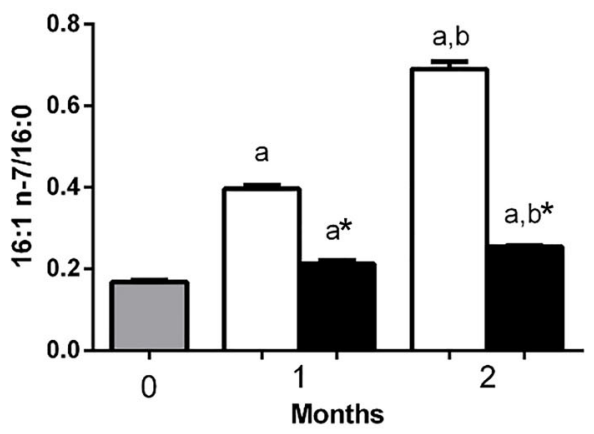

D6D

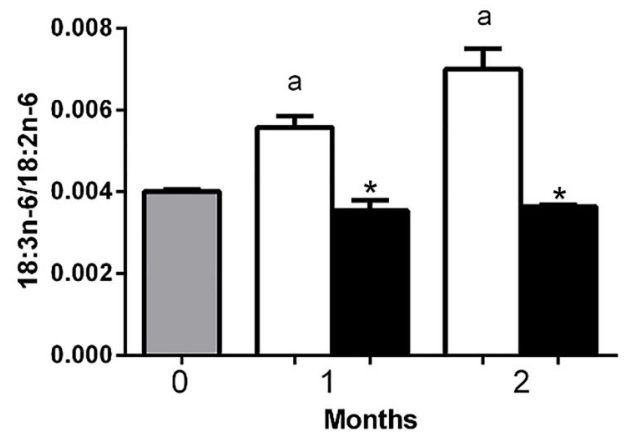

Elongase

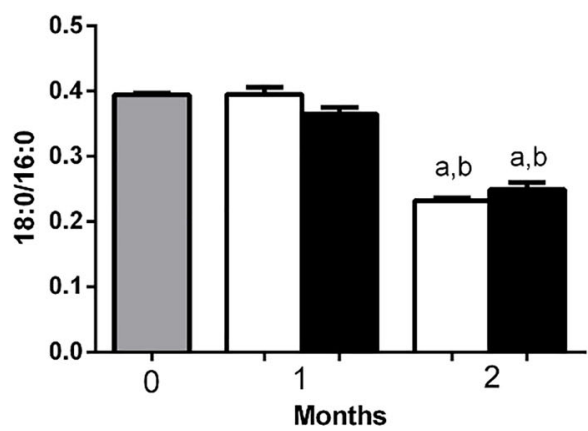

DNL

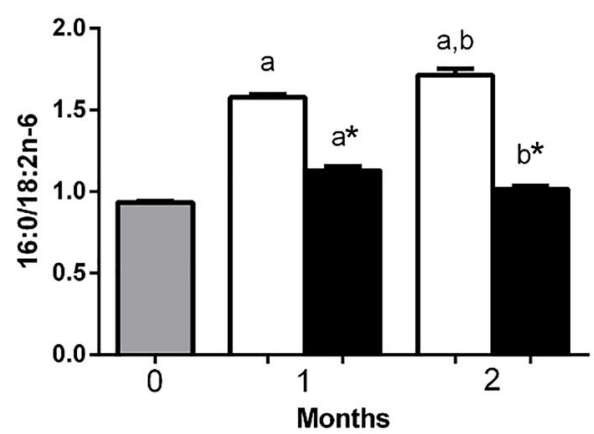

Figure 7. Activities of SCD-1, D6D, elongase 6, and de novo lipogenesis (DNL) in the gastrocnemius muscle from mice at 0 (before starting the diets, gray bars) or fed with a high-carbohydrate diet (HCD, white bars) or high-fat diet (HFD, black bars) 1 or 2 months. Results are reported as means $\pm \mathrm{SE}$. ${ }^{*} \mathrm{P}<0.05$ compared to HCD group (Student's $t$-test). ${ }^{\mathrm{a}} \mathrm{P}<0.05$ compared to time 0 ; ${ }^{\text {b }} \mathrm{P}<0.05$ compared to 1 month (one-way ANOVA). SCD-1: stearoyl-CoA desaturase-1; $\Delta-6$ desaturase (D6D), DNL: de novo lipogenesis.

\section{Inflammation assessment}

The HCD group exhibited higher $(\mathrm{P}<0.05)$ MPO activity after 2 months of diet interventions. The values (Ab $460 \mathrm{~nm}$ ) reported as means \pm SE of 8-10 mice per group were: $0.40 \pm 0.02$ in the HCD group and $0.33 \pm 0.01$ in the HFD group. IL- 6 mRNA expression was increased $(P<0.05)$ in the HCD group compared to the HFD group after 2 months (Figure 8).

\section{Discussion}

Macronutrient composition modulates FA deposition and inflammation in different tissues such as liver (14), brain (15), adipose tissue $(20,21)$, and serum $(17,21)$.

The HFD group had the same final body weight as the HCD group, despite lower caloric intake. These results are in agreement with other studies indicating that calories in the form of fat favor higher fat deposition compared with a high-carbohydrate diet $(8,20)$.

The HFD group exhibited lower DNL activity, indicating a lower conversion of carbohydrates to lipids. However, the HFD group had a higher content of SFAs, MUFAs, PUFAs, and total lipid accumulation in the gastrocnemius

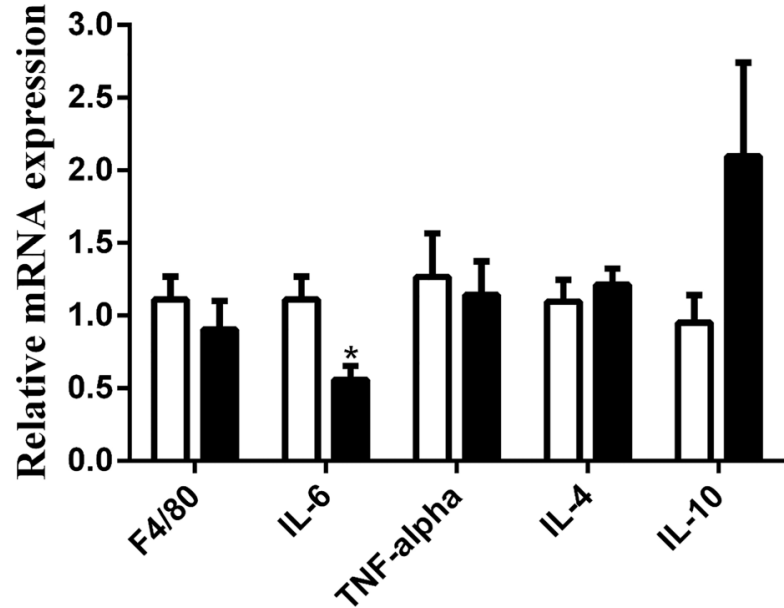

Figure 8. mRNA expression in gastrocnemius muscle of mice fed with either high-carbohydrate diet (HCD group, white bars) or highfat diet (HFD group, black bars) for 2 months. $\beta 2$-microglobulin ( $\beta 2 \mathrm{~m}$ ) was used as the housekeeping gene. Results are reported as means \pm SE $(n=8-10)$. ${ }^{*} P<0.05$ compared to the HCD group (Student's $t$-test). TNF- $\alpha$ : tumor necrosis factor alpha; IL: interleukin. 
muscle, which reflects at least in part the five times higher quantity of FA in this diet compared with HCD (14). Therefore, the higher DNL found in the gastrocnemius muscle of the HCD group did not compensate for the higher lipid intake of the HFD group. High fat intake may increase lipoprotein lipase activity and triacylglycerol accumulation (22).

SCD-1 activity was elevated in HCD mice, as reported by others in skeletal muscle (23). This higher activity leads to the generation of palmitoleic acid $(16: 1 n-7)$, vaccenic acid (18:1n-7), and oleic acid (18:1n-9) (24).

The deposition of PUFAs strongly correlates with dietary FA availability as reported for linoleic acid (18:2n-6) and $\alpha$-linolenic acid (18:3n-3) (25). This agrees with the higher quantities of linoleic acid (18:2n-6) and $\alpha$-linolenic acid $(18: 3 n-3)$ found in the gastrocnemius muscle of the HFD mice.

Arachidonic acid (20:4n-6) is a precursor of proinflammatory prostaglandins, thromboxanes, and leukotrienes (26), whereas docosahexaenoic acid (DHA, 22: $6 n-3)$ is a precursor of anti-inflammatory mediators (27). Therefore, the increased arachidonic acid (20:4n-6) and the reduced docosahexaenoic acid (DHA, 22:6n-3) contents in the HCD group after 2 months of diet interventions suggested a higher inflammatory state in the muscle of this group. In agreement with this postulation, inflammation was more significant in the HCD group, which showed higher IL-6 expression and MPO activity after 2 months of diet interventions. The skeletal muscle itself and muscle tissue infiltrating inflammatory cells produce IL-6, as observed in type 2 diabetes and inflammatory myopathies (28). MPO activity is a marker of neutrophil infiltration, which is related to cell regeneration activity after tissue injury (29). Both IL-6 expression and MPO activity are predictors of obesity-associated morbidities, pre-diabetes, and cardiovascular diseases (30).

\section{References}

1. Bergman BC, Perreault L, Strauss A, Bacon S, Kerege A, Harrison $\mathrm{K}$, et al. Intramuscular triglyceride synthesis: importance in partitioning muscle lipids in humans. Am J Physiol Endocrinol Metab 2018; 314: E152-E164, doi: 10.1152/ajpendo.00142.2017.

2. Vettor R, Milan G, Franzin C, Sanna M, De Coppi P, Rizzuto $\mathrm{R}$, et al. The origin of intermuscular adipose tissue and its pathophysiological implications. Am J Physiol Endocrinol Metab 2009; 297: E987-E998, doi: 10.1152/ajpendo.00229. 2009.

3. Manco M, Greco AV, Capristo E, Gniuli D, De Gaetano A, Gasbarrini G. Insulin resistance directly correlates with increased saturated fatty acids in skeletal muscle triglycerides. Metabolism 2000; 49: 220-224, doi: 10.1016/S00260495(00)91377-5.

4. Araújo $E P$, De Souza $C T$, Ueno $M$, Cintra DE, Bertolo MB, Carvalheira JB, et al. Infliximab restores glucose homeostasis in an animal model of diet-induced obesity and
The higher inflammation contrasted with the lower muscle FA accumulation in the HCD group. In our previous work, we found a higher accumulation of FA and inflammation intensity in the liver of the HCD group compared with the HFD group, as a consequence of more elevated DNL associated with high-carbohydrate ingestion (14).

The SFA/n-3 PUFA ratio was higher in skeletal muscle of the HCD group, whereas the $n-6 / n-3$ PUFA ratio remained unchanged. These results are in agreement with Rasic-Milutinovic et al. (31) who reported that the SFA/n-3 PUFA ratio is a better indicator of inflammation than the $n-6 / n-3$ PUFA ratio, considering the inflammatory properties of SFA and $n-6$ and the anti-inflammatory properties of $n-3$ PUFAs $(25,27,32)$.

The short period (2 months) of the diet interventions was a limitation of this study. Long-term studies are necessary to clarify the development of skeletal muscle inflammation and fatty acid composition in Swiss mice fed a high-carbohydrate or a high-fat diet.

In conclusion, our results demonstrated the importance of differentiating the roles played by macronutrient composition and lipid deposition. Furthermore, contrasting with the well-established idea that increased lipid deposition is associated with more intense inflammation, the HCD was more potent to induce skeletal muscle inflammation than the HFD, regardless of the lower lipid accumulation.

\section{Acknowledgments}

The following research agencies supported this study: CAPES (Grant number 88882.448882/2019-01), CNPq/ PRONEX/Araucária Foundation (Grant number 249/13), and Fundação de Amparo à Pesquisa do Estado de São Paulo-FAPESP (Grant number 2018/09868-7).

diabetes. Endocrinology 2007; 148: 5991-5997, doi: 10.1210/ en.2007-0132.

5. Hariri N, Thibault L. High-fat diet-induced obesity in animal models. Nutr Res Rev 2010; 23: 270-299, doi: 10.1017/ S0954422410000168.

6. Ochiai M, Matsuo T. Effects of short-term dietary change from high-carbohydrate diet to high-fat diet on storage, utilization, and fatty acid composition of rat muscle triglyceride during swimming exercise. J Clin Biochem Nutr 2009; 44: 168-177, doi: 10.3164/jcbn.08-237.

7. Bonen A, Parolin ML, Steinberg GR, Calles-Escandon J, Tandon NN, Glatz JF, et al. Triacylglycerol accumulation in human obesity and type 2 diabetes is associated with increased rates of skeletal muscle fatty acid transport and increased sarcolemmal FAT/CD36. Faseb J 2004; 21: 1-2.

8. Masi LN, Martins AR, Crisma AR, do Amaral CL, Davanso MR, Serdan TDA, et al. Combination of a high-fat diet with sweetened condensed milk exacerbates inflammation and 
insulin resistance induced by each separately in mice. Sci Rep 2017; 7: 3937, doi: 10.1038/s41598-017-04308-1.

9. Antunes MM, De Almeida-Souza CB, Godoy G, Crisma AR, Masi LN, Curi R, et al. Adipose tissue is less responsive to food restriction anti-inflammatory effects than liver, muscle, and brain in mice. Braz J Med Biol Res 2019; 52: e8150, doi: 10.1590/1414-431x20188150.

10. Andersson A, Nälsén C, Tengblad S, Vessby B. Fatty acid composition of skeletal muscle reflects dietary fat composition in humans. Am J Clin Nutr 2002; 76: 1222-1229, doi: 10.1093/ajcn/76.6.1222.

11. Turner N, Lee JS, Bruce CR, Mitchell TW, Else PL, Hulbert AJ, et al. Greater effect of diet than exercise training on the fatty acid profile of rat skeletal muscle. J Appl Physiol 2004; 96: 974-980, doi: 10.1152/japplphysiol.01003.2003.

12. Campbell TL, Mitchell AS, McMillan EM, Bloemberg D, Pavlov D, Messa I, et al. High-fat feeding does not induce an autophagic or apoptotic phenotype in female rat skeletal muscle. Exp Biol Med 2015; 240: 657-668, doi: 10.1177/ 1535370214557223.

13. Zhou W, Davis EA, Dailey MJ. Obesity, independent of diet, drives lasting effects on intestinal epithelial stem cell proliferation in mice. Exp Biol Med 2018; 243: 826-835, doi: $10.1177 / 1535370218777762$.

14. da Silva-Santi L, Antunes MM, Caparroz-Assef SM, Carbonera F, Masi LN, Curi R, et al. Liver fatty acid composition and inflammation in mice fed with high-carbohydrate diet or high-fat diet. Nutrients 2016; 8. pii: E682, doi: 10.3390/ nu8110682.

15. Gimenez da Silva-Santi L, Masetto Antunes M, Mori MA, Biesdorf de Almeida-Souza C, Vergílio Visentainer J, Carbonera $F$, et al. Brain fatty acid composition and inflammation in mice fed with high-carbohydrate diet or high-fat diet. Nutrients. 2018; 10. pii: E1277, doi: 10.3390/nu10091277.

16. Barrena HC, Schiavon FP, Cararra MA, Marques Ade CR, Schamber CR, Curi R, et al. Effect of linseed oil and macadamia oil on metabolic changes induced by high-fat diet in mice. Cell Biochem Funct 2014; 32: 333-340, doi: 10.1002/cbf.3018.

17. de Almeida-Souza CB, Antunes MM, Godoy G, Schamber CR, Silva MARCP, Bazotte RB. Interleukin-12 as a biomarker of the beneficial effects of food restriction in mice receiving high fat diet or high carbohydrate diet. Braz J Med Biol Res 2018; 51: e7900, doi: 10.1590/1414-431x20187900.

18. Bligh EG, Dyer WJ. A rapid method of total lipid extraction and purification. Can J Biochem Physiol 1959; 37: 911-917, doi: 10.1139/059-099.

19. Santos OO, Montanher PF, Bonafé EG, Prado IN, Maruyama SA, Matsushita M, et al. A simple, fast and efficient method for transesterification of fatty acids in foods assisted by ultrasound energy. J Braz Chem Soc 2014; 25: 17121719, doi: 10.5935/0103-5053.20140166.

20. Hu S, Wang L, Yang D, Li L, Togo J, Wu Y, et al. Dietary fat, but not protein or carbohydrate, regulates energy intake and causes adiposity in mice. Cell Metab 2018; 23: 415-431, doi: 10.1016/j.cmet.2018.06.010.

21. Wolk A, Furuheim M, Vessby B. Fatty acid composition of adipose tissue and serum lipids are valid biological markers of dairy fat intake in men. $J$ Nutr 2001; 131: 828-833, doi: 10.1093/jn/131.3.828.

22. Kiens B, Essen-Gustavsson B, Gad P, Lithell H. Lipoprotein lipase activity and intramuscular triglyceride stores after long-term high-fat and high-carbohydrate diets in physically trained men. Clin Physiol 1987; 7: 1-9, doi: 10.1111/j.1475097X.1987.tb00628.x.

23. Houdali B, Wahl HG, Kresi M, Nguyen V, Haap M, Machicao $F$, et al. Glucose oversupply increases $\Delta 9$ desaturase expression and its metabolites in rat skeletal muscle. Diabetologia 2003; 46: 203-212, doi: 10.1007/ s00125-002-1015-2.

24. Drạg J, Goździalska A, Knapik-Czajka M, Gawędzka A, Gawlik K, Jaśkiewicz J. Effect of high carbohydrate diet on elongase and desaturase activity and accompanying gene expression in rat's liver. Genes Nutr 2017; 12: 2, doi: 10.1186/s12263-017-0551-9.

25. Simopoulos AP. An increase in the omega-6/omega-3 fatty acid ratio increases the risk for obesity. Nutrients 2016; 8: 128, doi: 10.3390/nu8030128.

26. Korotkova M, Lundberg IE. The skeletal muscle arachidonic acid cascade in health and inflammatory disease. Nat Rev Rheumatol 2014; 10: 295-303, doi: 10.1038/nrrheum. 2014.2.

27. Serhan CN. Novel eicosanoid and docosanoid mediators: Resolvins, docosatrienes, and neuroprotectins. Curr Opin Clin Nutr Metab Care 2005; 8: 115-121, doi: 10.1097/ 00075197-200503000-00003.

28. Järvinen $T A$, Järvinen $T L$, Kääriäinen $M$, Kalimo $H$, Järvinen M. Muscle injuries: biology and treatment. Am J Sports Med 2005; 33: 745-764, doi: 10.1177/0363546505274714.

29. Faith $M$, Sukumaran $A$, Pulimood AB, Jacob M. How reliable an indicator of inflammation is myeloperoxidase activity? Clin Chim Acta 2008; 396: 23-25, doi: 10.1016/j.cca.2008. 06.016

30. Agarwal A, Hegde A, Yadav C, Ahmad A, Manjrekar PA, Srikantiah RM. Assessment of oxidative stress and inflammation in prediabetes-A hospital based cross-sectional study. Diabetes Metab Syndr 2016; 10: S123-S126, doi: 10.1016/j.dsx.2016.03.009.

31. Rasic-Milutinovic Z, Perunicic G, Pljesa S, Gluvic Z, Sobajic $S$, Djuric I. Effects of $n-3$ PUFAs supplementation on insulin resistance and inflammatory biomarkers in hemodialysis patients. Ren Fail 2009; 29: 321-329, doi: 10.1080/088602 20601184092.

32. Pillon NJ, Arane K, Bilan PJ, Chiu TT, Klip A. Muscle cells challenged with saturated fatty acids mount an autonomous inflammatory response that activates macrophages. Cell Commun Signal 2012; 10: 30, doi: 10.1186/1478-811X10-30. 